\title{
The Unveiling of the newly discovered Vela Supercluster *
}

\author{
Renée C. Kraan-Korteweg \\ Astrophysics, Cosmology and Gravity Centre, University of Cape Town, 7701 Rondebosch, \\ South Africa \\ E-mail: kraaneast.uct.ac.za
}

\section{Michelle E. Cluver}

Department of Physics, University of the Western Cape, Bellville, South Africa

\section{Maciej Bilicki}

Astrophysics, Cosmology and Gravity Centre, University of Cape Town, 7701 Rondebosch, South Africa \& Leiden Observatory, Leiden University, The Netherlands

\section{Matthew M. Colless}

Research School of Astronomy and Astrophysics, Australian National University, Canberra, Australia

\begin{abstract}
Multi-object spectroscopic observations of high density galaxy concentrations were obtained between 2012 and 2014 with SALT, using the multi-object spectrometer (MOS) of the Robert Stobie Spectograph (RSS) on SALT. The goal was to find the missing clusters in an earlier identified extended galaxy overdensity centered at $\sim 18000 \mathrm{~km} \mathrm{~s}^{-1}$ located at low Galactic latitudes in Vela. Reliable redshifts could be extracted for $\sim 80 \%$ of the targeted, highly-obscured galaxies. They were found to have an accuracy of the order of $\sigma \sim 150 \mathrm{~km} \mathrm{~s}^{-1}$. Of the 13 observed fields, ten revealed clear signatures of galaxy clusters. The majority of the clusters form part of the Vela overdensity. Their distribution also confirmed our suspicion that the Vela overdensity is even more extended and seems to straddle the Galactic Plane. Subsequent multi-fibre spectroscopy with AAOmega $+2 \mathrm{dF}$ on the Australian Telescope confirmed that these clusters are embedded in a gigantic overdensity of about $20^{\circ} \times 20^{\circ}$ on the sky. The overdensity and its clusters show strong similarities to massive superclusters such as the Shapley Supercluster. This previously unknown Vela Supercluster may well constitute an additional missing piece of the puzzle in solving the various contradictory (residual) bulk flow results given its location on the sky.
\end{abstract}

SALT Science Conference 2015

1-5 June 2015

Stellenbosch Institute of Advanced Study, South Africa

\footnotetext{
*Based on observations made with the Southern African Large Telescope (SALT)

† Speaker.
} 


\section{Introduction}

An inventory of the mass distribution in the local universe has for decades remained incomplete due to the obscuration of the Milky Way. This renders a derivation of the motion of the Local Group (LG), as evidenced by the dipole in the Cosmic Microwave Background ( $\left.\sim 613 \mathrm{~km} \mathrm{~s}^{-1}[6]\right)$, difficult. The direction and amplitude of the LG motion determined from the distribution of galaxies in redshift space or from peculiar velocity fields produce highly contentious results, e.g. $[8,5,10,2,18]$. Early discussions focused on the prominence of the Great Attractor at about 50 $\mathrm{Mpc} / \mathrm{h}$, e.g. [14]. But later derivations consistently find flows that arise from much larger scales $(\gtrsim 100 \mathrm{Mpc}[3,7])$. In addition, the recent results from the 6dFGS (6dF Galaxy Survey) peculiar velocity survey find a large residual bulk flow of $273 \mathrm{~km} \mathrm{~s}^{-1}$, suggestive of a significant mass concentration beyond their probed volume [16]. This is also seen in the watershed visualisations beyond the Laniakea Supercluster [19]. All of these derivations point to a considerable mass excess at large distances $(~ Z 160 \mathrm{Mpc} / \mathrm{h}$ ) close to the Zone of Avoidance (ZOA) toward the Vela constellation.

Intriguingly, it is exactly in this part of the ZOA that our longterm efforts of uncovering the large-scale galaxy distribution behind the Milky Way provided indications for a substantial overdensity at $\sim 180 \mathrm{Mpc} / \mathrm{h}$. It first became apparent in the distribution of the 350 redshifts obtained with the $6 \mathrm{dF}$ multifibre spectrograph of the UK Schmidt telescope covering two contiguous fields $\left(\sim 10^{\circ} \times 5^{\circ}\right)$ in Vela. We therefore launched a larger observing campaign to assess the full extent of the Vela overdensity. In a first step, we used SALT, the Southern African Large Telescope $(10 \mathrm{~m})$, to cover the highest galaxy density peaks and to identify the central clusters that are predicted by structure formation theory to reside at the bottom of such deep potential mass wells. In a second step, we employed AAOmega $+2 \mathrm{dF}$ of the Australian Telescope $(3.9 \mathrm{~m})$ to cover a much larger areal region to try to establish the overall extent of the Vela overdensity, which we suspect to be significantly larger than previously surveyed.

In the following chapter, we will present the newly acquired optical spectroscopic results. Pointings were distributed over an area a $40^{\circ} \times 20^{\circ}$ centered on the Galactic Plane at $(\ell, b) \sim$ $\left.275^{\circ}, 0^{\circ}\right)$ and encompassing the $6 \mathrm{dF}$ overdensity. The spectroscopic target list is based on the deep optical searches for partially obscured galaxies behind the southern Milky Way $\left(|b| \lesssim 10^{\circ}[11,20]\right.$ see also [13]), combined with near-infrared selected galaxies listed in 2MASX [9]. In view of the focus of this conference, major emphasis is given to the SALT observations.

\section{Optical spectroscopic results}

\subsection{SALT observations}

The multi-object spectrometer (MOS) can have up to 30 slitlets over a small field of view (8 arcmin). It is ideal for the highest density peaks in the ZOA galaxy distribution, which likely are clusters. The SALT-MOS cluster target fields are selected from our deep optical ZOA catalogs combined with 2MASX. The apparent, not for extinction corrected magnitudes range from $17.2-19.5$ in the $B$-band, respectively $11^{\mathrm{m}}-14^{\mathrm{m}}$ in the 2 MASX $K_{s}$-band. Accounting for the foreground obscuration, this conforms to about an $\mathrm{M}^{*}$-galaxy at the Vela overdensity distance range. With this luminosity constraint we will sample only the high end of the luminosity function of prospective Vela clusters. It also results in a relatively low number $(6-12)$ of slitlets for the 
target galaxies with an additional set of fibres dedicated to bright stars in the field for alignment and astrometry calibration. Nevertheless, as shown below, this was sufficient to identify which of the clumps were due to clusters (the majority).

Observing time with SALT was allocated in three semesters, 2012-1, 2013-1 and 2013-2. The first semester was regarded as pilot project. It was the first semester that RSS with MOS was available for science observations. In our first semester, we were allocated 10 fields of P2 grey time. For our chosen set-up (PG900 grating, $2^{\prime \prime}$ slits, $15^{\circ}$ VPH angle, $\sim 1.5-2.0^{\prime \prime}$ seeing), the RSS simulator suggested exposures time of $750 \mathrm{sec}(2 \times 375 \mathrm{sec})$ per MOS field for the acquisition of spectra with sufficient $\mathrm{S} / \mathrm{N}$ for redshift extraction. Including the overhead, $1700 \mathrm{sec}$ of SALT time were required per field.

In that first semester, 4 fields were observed. The raw data was processed with the basic PYSALT pipeline to produce image cubes and filled headers that could then be further reduced and analyzed using a suite of MOS-custom software tools. These reductions include individual slitmask (geometric) extraction, bias subtraction, basic wavelength calibration using Argon spectra, cosmic ray cleaning. More advanced processes included background subtraction using the full slitlet length (outside of the target galaxy emission), wavelength distortion correction using strong airglow lines and a 2-D cubic polynomial, and finally combining repeated observations to produce the best $\mathrm{S} / \mathrm{N}$ spatial-dispersion images. Collapsing to 1-D spectra, we then fit emission line and absorption line templates to determine the redshift.

Because most of the targets are 2MASX sources, the galaxies are early types or early spirals and tend to have absorption-line spectra. Conspicuous are the $\mathrm{Mg}(517.5 \mathrm{~nm})$ and $\mathrm{Na}(589.2 \mathrm{~nm})$ bands. A smaller fraction of galaxies have emission lines, notably the $\mathrm{H} \beta(486.1 \mathrm{~nm})$, [OIII] doublet at $\sim 500 \mathrm{~nm}$ and the $\mathrm{H} \alpha(656.3 \mathrm{~nm})$. Cross-correlating with these major lines, the redshift was determined for a small fraction of the targeted galaxies with an accuracy of a few percent (limited by the formidable wavelength distortion corrections); and later (see below), increasing the observing time and improving our distortion correction methods, we were able to extract redshifts for most targets with acceptable accuracy.

Figure 1 shows an absorption and an emission line spectrum of 2 galaxies observed in this run. Between 5-9 redshifts could be extracted in 3 out of the 4 observed fields. For the fourth field (Vela 8), only 1 spectrum led to a redshift. The 3 fields with multiple redshifts had clear galaxy cluster signatures (notable 'finger of god' distortion), 2 of them at the approximate Vela overdensity redshift range. This demonstrated that the science goal of the proposal could be achieved. However, the resulting spectra were noisier than expected due to the throughput problem. To extract science quality spectra for all of the targeted galaxies (fainter or low surface brightness galaxies, and galaxies at higher extinction levels), we increased the exposure times by a factor 1.5.

In our second run (2013-1), P1 time was allocated to 4 fields. All were observed, this time with an exposure on target this time of $1100 \mathrm{sec}$. Two of them yielded excellent results, and unveiled 2 more clusters in the Vela overdensity, one did provide a few random redshifts (Vela 7), hence was clearly not a cluster. The other (Vela 6) had a number of unusual spectra, where possibly something went amiss with the pointing or calibration. Although 3 of the 4 extracted redshifts put them in the Vela overdensity the data is insufficient to draw conclusion about whether this field marks a cluster.

In our third run (2013-2), 2 fields were allocated with P2 and 8 fields with P3 priority, resulting in a total of 6 observed fields that got observed. Exposure times ranged from $1100 \mathrm{sec}$ to $2100 \mathrm{sec}$. 

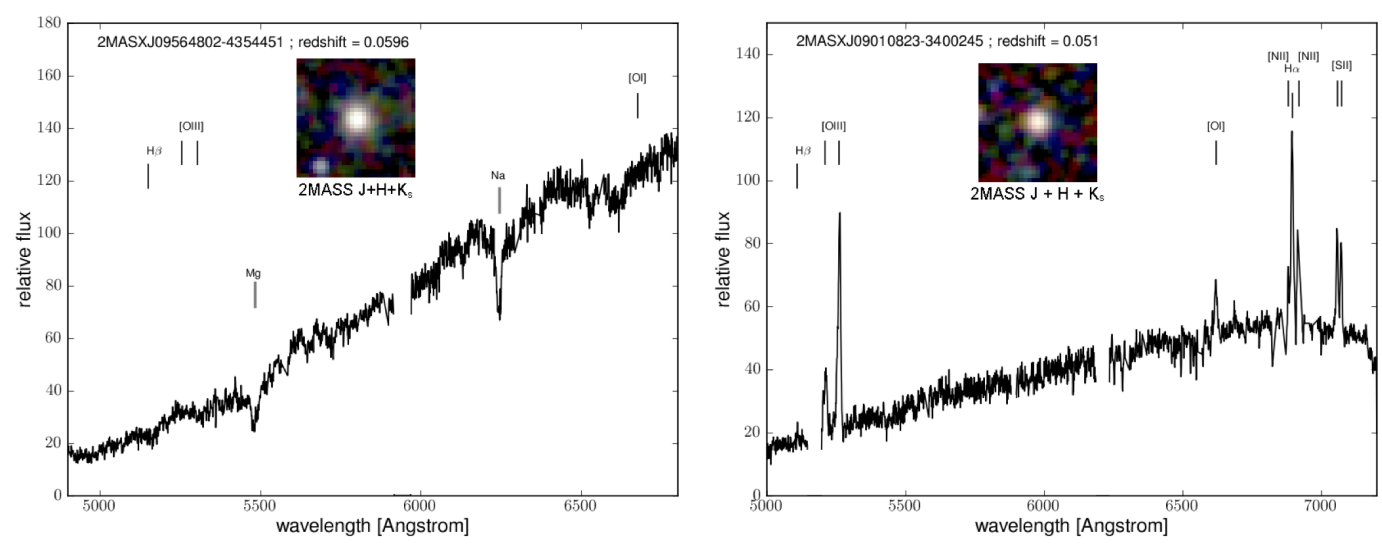

Figure 1: Absorption and emission line spectra of 2 galaxies observed in the first SALT observing run (2012-1) using MOS of the RSS for a total of $750 \mathrm{sec}$ on source. The galaxies are at intermediate extinction $\left(A_{B}=0 .{ }^{\mathrm{m}} 79\right.$ (left) and $1 .{ }^{\mathrm{m}} 39$ (right)) with magnitudes of $B_{J}=19{ }^{\mathrm{m}} 0, K_{S}=13{ }^{\mathrm{m}} 28$ and $B_{J}=17{ }^{\mathrm{m}} 5, K_{s}=12 \mathrm{~m} \cdot 51$ respectively. To achieve adequate $\mathrm{S} / \mathrm{N}$ spectra of fainter galaxies, and galaxies deeper in the ZOA, we increased the exposure times by a factor of 1.5 to 3.0 in subsequent semesters.

The data overall were very good, except for the repeat observation of Vela 8 with a longer exposure. Although more redshifts could be extracted only one was regarded as science quality.

A summary of the observed fields is given in Table 1, in order of the observing date. It lists our internal Vela cluster name, the Galactic coordinates, the optical obscuration $A_{B}$ at the center of the field, the observing date, the on-target exposure time in seconds (excluding overhead of $\sim 950 \mathrm{sec}$ ), the number of slits per field, the number of extracted spectra, the number of high quality (HQ) redshifts, and approximate mean redshift when a signature of a cluster was found.

In summary, the observations were a great success. Of the 13 fields that were targeted (one field twice), 10 revealed clusters in the ZOA of which 8 were previously unknown. An assessment of the quality of the redshifts indicate that $78 \%$ were of high, respectively science quality. While most of these fields lie at intermediate latitudes, one cluster (Vela 3) was unveiled deep in the Galactic Plane $(b=0.9)$, where the galaxies are fully obscured in the optical (hence fainter than about $B_{J} \gtrsim 20^{\mathrm{m}}$ ). Nevertheless, redshifts could be retrieved with SALT of the 2MASX counterparts.

Science-wise the observations let to important new insights. An astounding 7 (possibly 8) of the 10 clusters were found to lie at the velocity range of the Vela overdensity, that is $\sim 18000 \mathrm{~km} \mathrm{~s}^{-1}$. Particularly interesting is that three mark clusters in the $6 \mathrm{dF}$ fields of the Vela overdensity. We thus found the previously lacking deep potential wells of that massive overdensity. Secondly, clusters at the Vela overdensity redshift were found both over a wider longitude range and on the opposite side of the Galactic Plane, giving substance to the suspicion that this overdensity could be vast in size. As such it was a crucial point in our motivation to gain observing time with the $2 \mathrm{dF}+\mathrm{AAOmega}$ of the AT to map the smoother less dense galaxy overdensity over this much larger extent of the suspected overdensity.

Before we turn to the AAOmega observations, it should be noted that some of the AAOmega fields were positioned around the newly identified Vela clusters. This resulted in an overlap of 31 redshifts obtained with both SALT and AAOmega and allowed for a quantification of the accu- 


\begin{tabular}{|c|c|c|c|c|c|c|c|c|c|}
\hline ID & $\begin{array}{r}\text { central } \ell \\
\circ\end{array}$ & $\begin{array}{r}\text { central } b \\
\circ\end{array}$ & $\begin{array}{r}A_{\mathrm{B}} \\
\mathrm{mag}\end{array}$ & Obs Date & $\begin{array}{r}\text { Exp Time } \\
\text { sec }\end{array}$ & $\begin{array}{r}\text { slits } \\
\mathrm{N}\end{array}$ & $\begin{array}{r}\text { spectra } \\
\mathrm{N}\end{array}$ & $\begin{array}{r}\mathrm{HQ} z \\
\mathrm{~N}\end{array}$ & $\begin{array}{c}<z> \\
\mathrm{km} \mathrm{s}^{-1}\end{array}$ \\
\hline V04 & 260.0 & +8.1 & 1.60 & 14.06 .2012 & 750 & 6 & 5 & 5 & 12000 \\
\hline V08 & 258.1 & +8.1 & 1.55 & 15.06 .2012 & 750 & 6 & 2 & 1 & various \\
\hline V05 & 272.7 & +8.4 & 0.92 & 16.06 .2012 & 750 & 11 & 10 & 9 & 18000 \\
\hline V11 & 272.5 & +8.4 & 0.98 & 16.06 .2012 & 750 & 11 & 9 & 5 & 18000 \\
\hline V01 & 264.7 & -8.6 & 1.42 & 14.05 .2013 & 1100 & 12 & 10 & 7 & 18000 \\
\hline V06 & 264.4 & -9.1 & 1.05 & 22.10 .2013 & 1100 & 12 & 4 & 4 & 18000 \\
\hline V07 & 247.6 & -8.6 & 1.31 & 22.12 .2013 & 1100 & 8 & 4 & 3 & various \\
\hline CZ2 & 272.1 & -11.5 & 1.32 & 28.10 .2013 & 1100 & 12 & 11 & 11 & 18000 \\
\hline V16 & 264.4 & -8.9 & 1.07 & 23.12.2013 & 1200 & 7 & 3 & 1 & 18000 \\
\hline V02 & 246.6 & -9.2 & 0.93 & 20.01 .2014 & 1100 & 9 & 8 & 6 & 9000 \\
\hline V08 & 258.1 & +8.1 & 1.55 & 20.01 .2014 & 2100 & 6 & 2 & 1 & repeat \\
\hline V12 & 271.8 & +7.6 & 1.03 & 05.02 .1014 & 1200 & 8 & 7 & 6 & 18000 \\
\hline $\mathrm{CZ1}$ & 271.6 & -12.5 & 1.40 & 20.03 .2014 & 2100 & 10 & 8 & 7 & 18000 \\
\hline V03 & 251.5 & +0.9 & 2.24 & 21.03 .2014 & 1950 & 8 & 7 & 4 & 12000 \\
\hline
\end{tabular}

Table 1: Characteristics of the SALT MOS fields and results.

racy of the SALT spectra because the characteristics of the $2 \mathrm{dF}+\mathrm{AAOmega}$ are well known. For instance, the GAMA ${ }^{1}$ survey finds typical values of $\sigma \sim 100 \mathrm{~km} \mathrm{~s}^{-1}$ [1]. The dispersion between SALT and $2 \mathrm{dF}+\mathrm{AAOmega}$ for the 31 crossmatches is of the order of $\sim 200 \mathrm{~km} \mathrm{~s}^{-1}$. The SALT exposure times ranged from 12-20min compared 60 - 90min with AAOmega, hence balancing the difference in aperture quite well. This suggests that the errors of the SALT spectroscopy are slightly larger than the AAOmega spectra.

\subsection{AAOmega observations}

In 2014, we were allocated 6 nights of observing time with the 2dF+AAOmega spectrograph on the $3.9 \mathrm{~m}$ telescope of the Australian Astronomical Observatory (AAO) in 2014 $4^{2}$ This is an optimal instrument to survey large areas of sky with its 2-degree field of view within which up to 392 fibres can be positioned for each exposure. The 25 fields observed with AAOmega were spread over $\sim 40^{\circ}$ in longitude on either side of the Galactic Plane $\left(|b| \lesssim 10^{\circ}\right)$. The survey footprint is not contiguous in longitude. This was part of a sparse-sampling strategy to cover as wide an area as possible in the allocated observing period. Moreover, most of the pointings are towards intermediate latitudes $\left(|b| \sim 4-10.5^{\circ}\right)$ to optimise the use of the multi-fibre instrument and mitigate Galactic absorption at these wavelengths. The AAOmega observations led to over 4162 new science-quality redshifts. Further details on these observations will be given elsewhere.

\subsection{Overall results}

Combining the AAOmega and SALT redshift data with our earlier unpublished data, we have

\footnotetext{
${ }^{1}$ http://www.gama-survey.org/

${ }^{2}$ This observing time was originally allocated in early 2013 , just as a major bushfire swept over the observatory. Fortunately the telescope survived, and the observing was re-scheduled
} 
close to 4500 new redshifts in the ZOA survey area $\left(\ell \sim 285^{\circ}-245^{\circ}, b \lesssim \pm 10^{\circ}\right)$, where only a few were known beforehand. In the following, we will present some of the preliminary results. It should be kept in mind that the area remains sparsely sampled and that we have no redshifts at all for the most obscured part of the ZOA, i.e. for the latitude range of about $b \lesssim \pm 5^{\circ}$.

A redshift histogram is displayed in Fig. 2 for $(\ell, b)=\left(285^{\circ}-255^{\circ} ; \pm 10^{\circ}\right)$, i.e. the region in which the supercluster appears prominently. The distribution shows a highly significant peak centered at $17000-19000 \mathrm{~km} \mathrm{~s}^{-1}$, with broad shoulders around that peak ranging from $15000-$ $24000 \mathrm{~km} \mathrm{~s}^{-1}$. The grey-shaded area marks the redshift distribution below the Plane. The Vela overdensity can be traced over $\Delta \ell \sim 25^{\circ}$ above, and $\Delta \ell \sim 20^{\circ}$ over the same longitude range below the Galactic plane. The fact that the overdensity is nearly equally prominent on either side of the Plane strongly suggests the structure to be contiguous across the Plane, making it also $\Delta b \sim$ $20^{\circ}$ wide in latitude, leading to a minimal extent of $20^{\circ} \times 20^{\circ}$. The Vela redshift distribution, respectively the prominence of its peak, is similar to that of the Shapley supercluster area [15], one of the best studied superclusters in the nearby Universe.

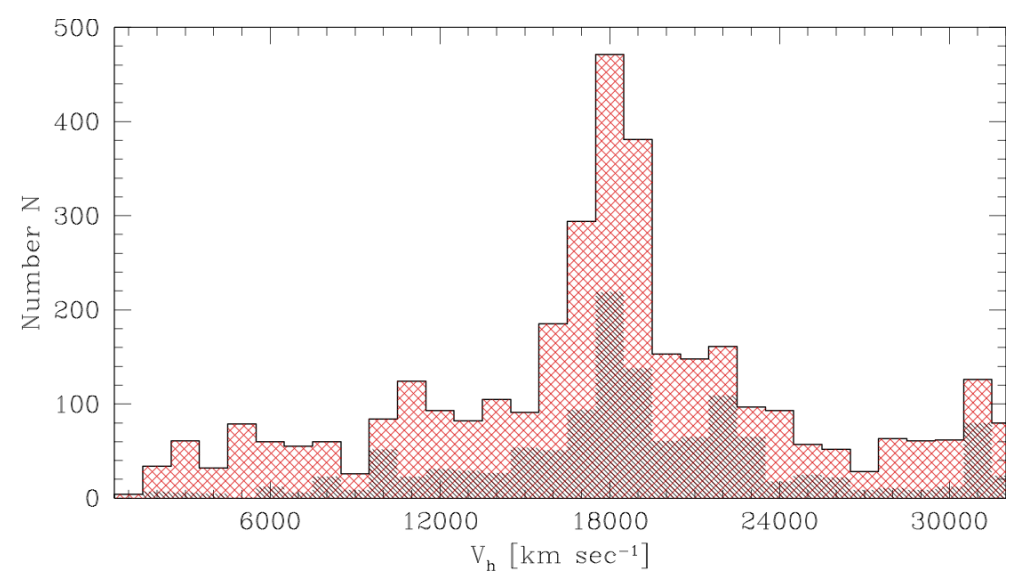

Figure 2: Velocity distribution in the direction of Vela overdensity for the ZOA redshift area delimited by $255^{\circ} \leq \ell \leq 285^{\circ} ;|b| \leq 10^{\circ}$ (red histogram). The grey shaded part are galaxies below the Galactic Plane only to emphasise the prominence of the overdensity on either side of the Plane. Note that only a few dozen redshifts were known in this area before.

Figure 3 displays the distribution of the same galaxies in a redshift cone along Galactic longitudes for the latitude range of $|b| \leq 10^{\circ}$. It is remarkable how well we can trace large-scale structures in the ZOA where hardly any data was available before. The redshift cone reveals the Vela overdensity to consist of an extended broad wall structure at about $18000 \mathrm{~km} \mathrm{~s}^{-1}$, with a second wall at slightly higher distances, which could well be merging around $\ell \sim 265^{\circ}$ with the main wall. Note that the gaps in the walls are artificial and caused by the still substantial gaps over the survey area. An algorithm identified numerous clusters of which 22 have a velocity dispersion of $>400 \mathrm{~km} \mathrm{~s}^{-1}$. This also compares favourably with Shapley, which contains 27 such clusters, and is (unlike the Vela SCL) fully sampled (whereas Vela survey has about $20 \%$ coverage).

Both the velocity histogram and the large numbers of clusters identified in the redshift data at hand show clear characteristics of a supercluster. Most superclusters are elongated, prolate structures [4], with clusters embedded in a wall-like structure which can show multi-branching of filaments and walls, or can even be composed of different superclusters. This also concurs with the 


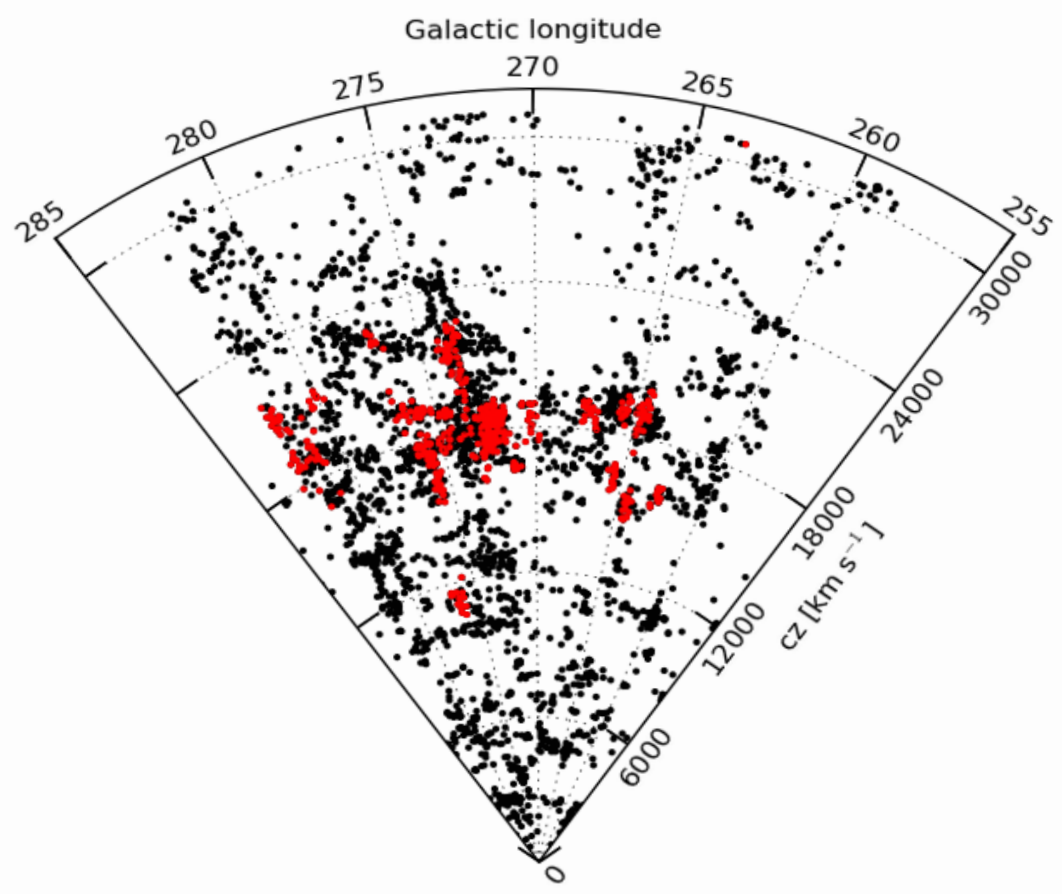

Figure 3: Wedge diagram out to velocities of $V_{\text {hel }}=32000 \mathrm{~km} \mathrm{~s}^{-1}$ for the same longitude and latitude range as in Fig. 2. The red dots mark the galaxies that belong to the identified galaxy clusters.

morphology of the broad but very extended walls traced in our redshift data. Even without data in the innermost part of the ZOA, all evidence seem to indicate that the Vela overdensity is a large supercluster in the nearby Universe. We henceforth call it the Vela Supercluster.

\section{Discussion and future plans}

Our observations in the ZOA have revealed a previously unknown supercluster in the constellation of Vela. It lies close in position and redshift to where many bulkflow derivations predict significant missing mass. Despite its extent is has eluded discovery so far, because of its location at low Galactic latitudes. Moreover, it lies just beyond the boundaries and volumes of current systematic "whole-sky" redshift and peculiar velocity surveys. As such it is highly likely that the Vela Supercluster will contribute to the LG motion and may well reduce the current misalignment of the derived dipoles based on galaxy surveys.

But a proper systematic mapping of the extent (volume) and overdensity (within this volume) of the Vela Supercluster is crucial. To make progress in this regard, further observations with AAOmega have been requested to minimally fill the gaps between the to date observed 25 fields and arrive at a systematic redshift coverage over the extent of the Vela Supercluster along the ZOA $\left(285^{\circ} \lesssim \ell \lesssim 255^{\circ}\right)$ for the latitude range over which we can reliably get redshifts $\left(|b| \approx 4^{\circ}-10^{\circ}\right.$, or $\left.A_{B} \lesssim 3 \mathrm{mag}\right)$. We have started a deep imaging program in the near-infrared of all potential clusters in the Vela SCL with the IRSF $(J H K)$ on the $1.4 \mathrm{~m}$ telescope at Sutherland allowing a full analysis of the clusters, their luminosity functions and individual masses. Follow-up SALT observations 
will be requested once this data is in hand. In regions of highest obscuration only $\mathrm{HI}$ line surveys can penetrate the ZOA $[12,17]$ and we envision to use MeerKAT ${ }^{3}$ in science commissioning time or with early science projects.

Acknowledgment: Financial support from the National Research Foundation and the University of Cape Town is gratefully acknowledged. Part of the observations reported in this paper were obtained with the Southern African Large Telescope (SALT) in 2012 and 2013. The respective proposal codes were 2012-1-RSA_POL-001, 2013-1-RSA_OTH-016 and 2013-2-RSA_OTH_001 with RKK being the Principle Investigator and TJ being the Principal Contact. Great thanks go to SALT astronomers Alexei Kniazev, Petri Vaisanen \& David Gilbank in their help in preparing the Phase II proposals and with the data reduction process (notably David Gilbank).

\section{References}

[1] Baldry I. K., Alpaslan, M. \& Bauer, A. E.et al., 2014, MNRAS 441, 2440

[2] Bilicki, M., Chodorowski, M. Jarrett, T. \& Mamon, G. A. 2011, ApJ 741, 31, 13pp

[3] Carrick, J., Turnbull, S. J., Lavaux, G. \& Hudson, M. J. 2015, MNRAS , 450, 317

[4] Einasto, M., Liivamägi, L. J., Saar, E. et al. 2011, A\&A, 532, 5, 20pp

[5] Erdogdu P., Huchra, J. P., Lahav, O. et al. 2006, MNRAS , 368, 1515

[6] Fixsen, D. J., Cheng, E. S., Gales, J. M. et al. 1996, ApJ 473, 576

[7] Hoffman, Y., Courtois, H. M. \& Tully, R. B. 2015, MNRAS , 449, 4494

[8] Hudson, M. J., Smith, R. J., Lucey, J. R. \& Branchini, E. 2004, MNRAS , 2004, 352, 61

[9] Jarrett, T. H., Chester, T., Cutri, R. et al. 2000, AJ , 119, 2498

[10] Kocevski, D. D. \& Ebeling, H. 2006, ApJ 645, 1043

[11] Kraan-Korteweg, R. C. 2000, A\&AS, 141, 123

[12] Kraan-Korteweg, R. C. 2005, Rev in Modern Ast, 18, 48

[13] Kraan-Korteweg, R. C., \& Lahav, O. 2000, A\&ARev, 10, 211

[14] Kraan-Korteweg, R. C., Woudt, P. A.; Cayatte, V. et al. 1996, Nature , 379, 519

[15] Proust, D., Quintana, H., Carrasco, E.R. et al. 2006, A\&A, 447, 133

[16] Springob, C. M., Magoulas, C., Colless, M. et al. 2014, MNRAS , 445, 2677

[17] Staveley-Smith, L., Kraan-Korteweg, R. C., Schroeder, A. C. et al. 2016, AJ , in press

[18] Turnbull, S. J., Hudson, M. J., Feldman, H. A. et al. 2012, MNRAS , 420, 447

[19] Tully, R. B., Courtois, H., Hoffman, Y., Pomarède, D. 2014, Nature , 513, 71

[20] von Maltitz, K. 2012, MSc thesis, University of Cape Town

\footnotetext{
${ }^{3}$ http://www.ska.ac.za/
} 